\title{
Obéir à Gavrinis
}

\section{Charles-Tanguy Le Roux}

\section{(QpenEdition \\ Journals}

Édition électronique

URL : https://journals.openedition.org/rao/7614

DOI : $10.4000 /$ rao.7614

ISSN : 1775-3732

\section{Éditeur}

Presses universitaires de Rennes

\section{Édition imprimée}

Date de publication : 21 décembre 2021

Pagination : 375-376

ISBN : 978-2-7535-8668-0

ISSN : 0767-709X

\section{Référence électronique}

Charles-Tanguy Le Roux, "Obéir à Gavrinis », Revue archéologique de l'Ouest [En ligne], 37 | 2021, mis en ligne le 21 décembre 2021, consulté le 11 octobre 2022. URL : http://journals.openedition.org/rao/ 7614 ; DOI : https://doi.org/10.4000/rao.7614 
ceinte et devient la porte monumentale de la cité. Dans le courant du haut Moyen Âge, l'amphithéâtre disparaît en tant qu'objet architectural à part entière sous l'effet du morcellement de son emprise, tout en restant un élément éminemment morphogène dans le sens où l'orientation de ses structures a conditionné l'organisation radioconcentrique du tissu urbain qui le recouvre au Moyen Âge et à la l'Époque moderne. En dépit d'une densification de l'espace associée à un renouvellement des constructions existantes, la nature de l'occupation reste stable dans la longue durée. Ces observations conduisent par ailleurs l'auteur à comparer la formation du tissu urbain implanté sur l'amphithéâtre de Tours avec vingt-sept villes européennes présentant des édifices comparables. Pour ce faire, il fait appel à une méthode de modélisation graphique inspirée de l'approche chorématique utilisée par les géographes, dans laquelle il distingue trois modèles théoriques, et dont les principes et les résultats sont exposés de façon plus détaillée dans un article de B. Lefebvre paru en 2011 dans la revue M@ppemonde. Dans le deuxième chapitre, il tente de préciser la nature de l'occupation interne de l'amphithéâtre entre le $v^{e}$ et le viII ${ }^{e}$ s. en s'appuyant sur les rares indices dont il dispose. C'est ainsi qu'il émet l'hypothèse d'un scénario en trois étapes. Il considère que le théâtre-forteresse aurait pu, dans un premier temps, appartenir au roi, avant d'être morcelé, selon un rythme qui reste à préciser et durant une période qui s'étend des $\mathrm{IX}^{\mathrm{e}}-\mathrm{X}^{\mathrm{e}} \mathrm{s}$. au début du XIII ${ }^{\mathrm{e}} \mathrm{s}$. entre l'archevêque, le chapitre et le roi, avant de devenir un quartier canonial à partir du XIII ${ }^{\mathrm{e}}$ s. Son fonctionnement est analysé dans un troisième chapitre, dans lequel B. Lefebvre compare notamment le cloître de Saint-Gatien avec celui de SaintMartin étudié par Hélène Noizet, et dans lequel il met en scène, dans leur rapport avec l'espace, le chapitre en tant qu'institution et les chanoines en tant que clercs, mais aussi comme individus.

Dans sa conclusion, B. Lefebvre revient sur les apports de son étude en soulignant sa portée historique, en insistant sur les dynamiques de la composition du tissu urbain du XIII ${ }^{e}$ s. au XVIII ${ }^{e}$ s. et en revenant sur les aspects méthodologiques.

Au terme de cette lecture, on peut considérer que les objectifs qui étaient de restituer les processus de transformation d'un édifice de spectacle antique en un quartier canonial ont été atteints avec maestria. C'est un nouveau pan de l'histoire de Tours qui est ici dévoilé, complétant opportunément les travaux de recherche menés sur la fabrique urbaine et sur la ville de Tours depuis les années 1990. Compte tenu des résultats obtenus et des méthodes mises en œuvre, cette étude, menée à la croisée de plusieurs champs disciplinaires, envisagée sur le temps long et sur une échelle rarement abordée, est appelée à devenir un modèle en matière d'analyse du processus de formation d'un tissu urbain. Elle constitue, en outre, une contribution originale à la connaissance des quartiers canoniaux du Moyen Âge à l'Époque moderne.

Yves Henigfeld

De Vial A. et Alfera G., 2011, Obéir à Gavrinis, portfolio de 14 doubles-feuillets $27 \times 32,5 \mathrm{~cm}$. Tirage limité (44 ex.), Le Quatre de Chiffre (atelier de l'auteur), Neauphle-leChâteau.

Depuis sa découverte en 1832, que n'a-t-on publié sur Gavrinis et sur l'ornementation unique de cette crypte dolménique! En près de deux siècles, les pages de textes - érudites ou moins savantes - se sont empilées; les relevés, moulages et photographies de cet art déroutant se sont eux aussi succédé, mais presque toujours dans un but analytique et interprétatif. Outre l'approche d'un Albert Gleizes (1939; Alibert, 1985) ou quelques pièces dans la production foisonnante de Jules Pressant autour des années 1970 (Puget, 1985), rares auront finalement été les artistes à s'imprégner de l'imagerie gavrinienne.

Alors qu'une nouvelle lecture scientifique de ce décor, à la lumière des techniques modernes du traitement d'image, est en cours sous la direction de Serge Cassen, c'est donc avec le plus grand plaisir que nous saluons le somptueux travail proposé conjointement par le poète Antoine de Vial et le peintre Gilles Alfera.

Partant du dernier relevé exhaustif disponible lors du lancement de ce travail (Shee-Twohig, 1981), ce dernier nous propose de revisiter les dix-huit principaux panneaux de Gavrinis sous forme d'eaux-fortes polychromes. Si les tracés restent scrupuleusement respectés, leur mise en teinte n'en suit que très librement la lecture " classique ». Cela peut dérouter le lecteur-archéologue mais ce n'est sans doute pas le moindre intérêt de la démarche. Ajoutons que la palette chromatique utilisée est ici tout en douceur, y compris par rapport à d'autres œuvres du même artiste (voir par exemple Alfera, 2009); ce n'est sans doute pas innocent et cela contribue à nous faire entrer dans son jeu.

Ce que l'on est convenu d'appeler l'art sacré tient une place importante dans l'œuvre de Gilles Alfera. Mais il s'agit cette fois de tout autre chose que de la tradition judéo-chrétienne qui lui est familière : l'artiste se confronte ici aux mystères de ce que j'ai naguère pu appeler un «Très Ancien Testament occidental ». De cette tradition perdue, seules de rares bribes - hélas devenues muettes - ont pu survivre aux millénaires sur les parois 
de quelques mégalithes privilégiés, et Gavrinis est sans doute un des meilleurs sites pour prendre conscience de cette richesse évanouie. Merci à Gilles Alfera de nous en proposer sa propre lecture, hors de toute fausse bienpensance archéologique (et "toute science dépassant " pour reprendre le vers de Jean de la Croix).

Pour chaque dalle, ce travail graphique inclut un aphorisme d'Antoine de Vial. Gilles Alfera a su faire épouser à l'un les courbes de l'autre, d'assez près pour qu'ils se valorisent mutuellement en une sorte de contrepoint visuel, mais sans que ces deux approches se fassent ombrage. Sur certaines planches, cela peut tenir de la gageure mais le pari est globalement fort bien gagné. Antoine de Vial signe également le texte poétique qui occupe les cinq premiers feuillets de l'ouvrage (travail qui, par ailleurs, a fait l'objet d'un pré-tirage en petit format : de Vial, 2012).

Éditée sur un magnifique papier bouffant, la série complète (les dix-huit planches et le texte) est présentée dans un somptueux portfolio de quatorze double-feuillets sous bồtage cartonné de $27,5 \times 35,5 \mathrm{~cm}$. Limité à quarante-quatre exemplaires, ce tirage d'auteur ${ }^{1}$ est également proposé en versions « allégées » de douze et six gravures, celles-ci pouvant aussi être obtenues à l'unité, faciles à encadrer. Sous une forme ou sous une autre, c'est là une bien belle idée de cadeau - en même temps qu'un beau sujet de réflexion pour tout amateur d'art comme d'archéologie (ce qui n'est nullement incompatible!).

Charles-Tanguy Le Roux

\section{Bibliographie}

Alfera G., 2009, Entre Ciel et Terre : la vision d'un peintre, Paris, Scriptoria, $62 \mathrm{p}$.

Gleizes A., 1939, "Tradition et Modernité ", L'Art et les artistes, ${ }^{\circ} 193$, p. 110.

Puget C. (dir.), 1985, Jules Paressant, Pont-Aven, Ville de Pont-Aven, catalogue d'exposition, $80 \mathrm{p}$.

De Vial A., 2012, Obéir à Gavrinis, Paris, Orizons, 54 p.

SheE-Twonig E., 1981, The megalithic Art of Western Europe,

Oxford, Clarendon Press, 259 p. + fig. \& pl.

1. 77 Grand-Rue, 78640 Neauphle-le-Château (gilles@alfera.org) ; aperçu sur le site [http://alfera.org/]. 